\title{
Effects of Sleep Disorders on Academic Achievement of Students at University Level
}

\author{
* Naila Iram, MS Scholar \\ ** Dr. Shamsa Aziz, Associate Professor \& Chairperson \\ *** Dr. Fouzia Ajmal, Assistant Professor (Corresponding Author)
}

\begin{abstract}
Sleep is a physiological process essential to humans for their normal functioning. Sleep habits and problems are also influenced by physical, mental, and environmental factors such as age, gender, job, lifestyle, emotional tension, and noise. The purpose of this study was to explore the effects of sleep disorders on students' academic achievement at International Islamic University Islamabad. The objectives of the study were to: find out the causes of sleep disorders among university students and explore the effect of sleep disorders on the academic achievement of students. This study was quantitative in nature and a survey was done for data collection. The sample of the study consisted of 80 students of the Faculty of Social Sciences suffering from sleep disorder and residing in hostels. Researchers used a self-developed three-point scale for data collection. The validity of the instrument was checked through experts' opinions while the reliability of the instruments was checked through Cronbach's Alpha. The collected data were scored, tabulated, and analyzed making use of descriptive and inferential statistics i.e. mean scores and t-test through Statistical Package for Social Sciences (SPSS 21). Results showed that late-night socializing and stress related to exams, assignments, and tests were the two top major causes of students' sleep disorders and there is a great effect of sleep disorders on the academic achievement of students.
\end{abstract}

Keywords: $\quad$ Sleep Disorder, Academic Achievement, Quantitative, University Students

Introduction

In the teaching and learning process sleeping plays a very important role in the mental processes as well as academic achievements of the students. In most of the research studies, it is presented that poor sleep quality, late sleeping, and wake up early in the morning and disturbed night sleep influence the behavior of the learner, and learning ability as well as students' academic achievements (Suchecki $\&$ Machado, 2009). Therefore, a sleep disorder is a common issue among learners. Sleep disorder disturbs the social and educational life and academic achievement of the students (Curcio, Ferrara \& Gennaro, 2006). Thus, one of the key requirements for learners is that the learners must be good physically, academically, and emotionally (Neinstein et al., 2008). Thus, sleep is a dynamic, action helping numerous utilities, for example, healing as well as development, memory alliance, \& uplifting practices. Sleep disorder might be the outcome of deficiency of arguments, mental and neurocognition (Curcio et al. 2006).

Moreover, a maximum shared consequence of insufficient or interrupted sleep is augmented by sleepiness throughout the whole day. Increased drowsiness throughout the whole day, outcomes in concentrated attentiveness, it can affect the neurocognitive dysfunction (Dimitriou \& Milton, 2015). According to this investigation, we intended to measure sleep disorders' influence on pupils' classroom performance as well as overall academic achievement at the university level. Sleep is a mental procedure important to human beings for their regular and efficient working. Sleep habits and problems related to sleep are also influenced by physical, psychological, and ecological features, for example, age, gender, job, life, emotive tightness \& noise etc. Every learner needs 7 hours of sleep every night (Zeek, Savoie, Song, Kennemur, Qian, Jungnickel, \& Westrick, 2015). Every child needs to sleep properly at least seven out of 24 hours because if children sleep properly it will impact positively on their health and psychologically as well (Suchecki \& Machado, 2009). Moreover, in sleeping, the mind conducts recollection amalgamation \& incorporation; and permits the mind to

\footnotetext{
* Department of Education International Islamic University, Islamabad Email: naila.msedu316@iiu.edu.pk

** Department of Education International Islamic University, Islamabad Email: shamsa.aziz@iiu.edu.pk

*** Department of Education International Islamic University, Islamabad Email: fouzia.ajmal@iiu.edu.pk
} 
improved procedure innovative involvements and information which escalations considerate and preservation.

Thus, in the learning process sleeping is very important for retention developments and academic achievement of the students. According to numerous researches not average sleeping or sleep at late hours, wake up early in the morning as well as disturbed night sleep influence performance, knowledge capability, and school achievement (Curcio, Ferrara \& Gennaro, 2006). The children who do not take average sleep in the whole day negatively affect the psychological, societal, educational, and academic achievements of the students. Thus, sleep disorders comprise an inclusive variety, from deteriorating sleeping trouble and awakening up regularly throughout the night, to the severe prime sleep sicknesses like disruptive sleep conditions (Anderson, Isser \& Taylor, 2009).

Conversely, other elements for example age \& gender of pupils, paternal profession, education, and married life, could also be linked with academic achievement. The occurrence of sleep disorders in every child is from eleven to $47 \%$. Thus, according to the research, the occurrence of sleep disorders has been described almost $25 \%$ in child, and $40 \%$ in youths. While maximum realistic researches have showed that children acquire almost 9 hours of sleep on average every night, numerous researches have revealed that $45 \%$ of child and students, sleep less than 8 hours every night (Wolfson \& Carskadon, 2003). Students of universities have experienced sleep issues; which might be the reason for their low academic achievement, well-being, and attitude (Yang, Wu, Hsieh, Liu, \& $\mathrm{Lu}, 2003)$. A mutual sleep issue in pupils is sleep deficiency and subsequent daylight drowsiness. Thus, genetic as well as societal issues contribute to deficiency. Furthermore, sleep might willingly forego because of societal influences or unwillingly condensed because of living in a piercing dwelling hall or home.

Asleep disorder can affect your complete health, protection as well as quality of life. Most of the signs of sleep disorders comprise extreme daylight sleepiness, unequal conscious or improved movement during sleep.

Student achievement measures the amount of academic content a learner learns in an unwavering amount of time. Every grade level has learning aims or instructional morals that educators are obligatory to teach. Student accomplishments will grow when quality teaching is cast-off to teach instructional principles.

\section{Statement of the Problem}

In the teaching-learning process sleep health plays a very significant role. Identifying the stability among university learners' sleep health can be thought-provoking. It might be a requirement to grow provision for university learners to well accomplish sleep encounters. It is significant to endorse the implication \& relevancy of sleep health and academic achievement. Conversely, it is indeterminate whether university learners observe sleep health as the further most important health tip that influences their academic achievement, as associated with other health aspects (such as, mutual cold, unhappiness, and association problems). The purpose of this study was to explore the effects of sleep disorders on students' academic achievement at International Islamic University Islamabad.

\section{Objectives}

The objectives of the study were to:

1. To find out the causes of sleep disorders among university students.

2. To explore the effect of sleep disorders on the academic achievement of students.

\section{Research Question \& Hypothesis}

The research question and hypotheses of the study were as follows:

Q.1. What are the major causes of sleep disorders among university students?

$\mathrm{H}_{\mathrm{o} 1} \quad$ There is no effect of sleep disorders on the academic achievement of students.

\section{Significance and Delimitations of the Study}

This study will be significant for students to know the aspects of sleeping that can affect their academic achievement. The findings of this study will enable teachers to be conscious on what is the reason for learner's lateness as well as to monitor them in their studies particularly in their academic or university achievement. This study will assist parents to perceive and display their child's sleeping period to avoid the habit of remaining conscious late at night.

This study was delimited to the residential BS students of the Faculty of Social Sciences International Islamic University Islamabad. 


\section{Literature Review}

Sleep is directly related to learning abilities (such as remembrance, consideration) (Hershner \& Chervin, 2014). According to neuroscientists, mental development occurs when one is sleeping (Smith, 2001). Distinguishing as well as appreciative the link amongst sleeping \& teaching-learning in neuroscience, consciousness, and neurobiology highpoints the significance of sleep health in learning; knowledge \& remembrance are two main features for academic achievement. Sleep health contains daylight attentiveness, sleep period, sleep competence, sleep judgment, plus sleep fulfillment (Buysse, 2014). Sleep disorders, mainly affect student academic achievement (Friedman, 2003). Pitiable sleep health might be preventing an individual's capabilities to study because of a deficiency of awareness, reduced recollection \& harmful distress. Indication has revealed that sleep health is the problem amongst university learners. Mutual sleep difficulties for university learners comprise sleep loss, hindered sleep, unequal sleep agenda, as well as daylight tiredness (Hershner \& Chervin, 2014). Immoral conduct as well as holding jobs that have late-night hours (such as, modification work) can affect harmful influences on university learners' sleep health. Primary, immoral habits, for example overwhelming caffeinated potions in the evening and twilight, consuming knowledge, earlier bed, could rise time to decrease sleeping, decline sleep hours, as well as growth daytime drowsiness (McKim, 2007).

Further immoral practice, imbibing liquor and pleasing sleep assistances to descent asleep, might be delay with the standard sleep phase, make sleep excellence inferior, grow occurrence of incubuses, as well as decline awareness throughout the day (Ebrahim, Shapiro, Williams, \& Fenwick, 2013). University learners' sleep health can also be compressed by functioning an evening shift, night shift, revolving change, splitting modification, and unequal programed. Change work can affect an unequal sleep timetable that carries to extreme daylight sleepiness, sleeplessness, condensed achievement, enlarged risk of effort coincidences, problems with individual associations, as well as destructive effects (for example, unhappiness). Therefore, the influence of sleeping difficulties on university learners' academic achievement has been planned for further than 40 years as well as diversified outcomes were originated. Comparatively high results were described by university learners who had an appropriate decent sleeping quality (Gomes, Tavares, \& Azevedo, 2011) thus, sleeping more than 9 hours (Kelly, Kelly, \& Clanton, 2001) as well as didn't postponement their sleep (Lack, 1986), and their result was meaningfully compressed in daylight tiredness, sleep period, as well as sleep inexpression) associations amongst sleep variables or results were not dependable. Rarely investigators establish that learner results were not meaningfully connected with sleep variables, for example, daylight sleepiness, disappointment with sleep, sleep hours, as well as sleep excellence, whereas numerous investigators described the result as destructively associated with sleep variables, for example, daylight sleepiness, hindered sleep time, sleep period, sleep agenda, as well as time wakeful before rising (Singleton, 2009).

Thus, sleep is a generally periodic state of awareness as well as body, depicts disaccustomed consciousness, abstemiously delayed corporeal capability, self-consciousness of practically every thoughtful influence, as well as diminished relations with situation (Bao, \& Yang, 2020). Hence, the significant and accustomed portion of everybody's lifespan, sideways with nourishment and implementation, is one of the three supports on which a vigorous \& cheerful life is founded, and we employ about a third of exists sleeping. Thus, sleep distinct that 'A period of contracted receptiveness to outer incentives, a decent excellence of sleep carries to a decent quality of academic achievement, when it originates to learners. So, this is particularly significant for youngsters that immobile in school. Offspring amongst the ages from 10 to 16 years, who have sleep-disordered consciousness, which comprises snuffling, sleeping apnea, as well as further kinds of sporadic consciousness throughout sleep, is most probable to have difficulties with consideration as well as education. It might lead to "important purposeful diminishing at university," the research writers inscribed. In another research, university learners who did not grow sufficient sleep had inferior results compare to those who did. "If you're demanding to encounter a limit, you're eager to detriment sleep," Dr. Torpey speaks, that it's simple as well as reoccurring sleep deficiency that damages teaching and learning process.

The Global Organization of Sleep Disorders classifies kinds of sleep disorders: dyssomnias, parasomnias, in addition to remedial/emotional illnesses. The effect of sleep loss on academic achievement may be understated. 50 university scholars, disadvantaged of sleep for Yang, et al. 
(2003) nightly, were then examined to resolve math adding issues. Subsequently, sleep damage; contributors were further probable to indicate calmer difficulties to resolve. Reisi et al. (2017) conducted a study in Iran for exploring the effect of sleep disorder on students' academic performance and found a negative effect of sleep disorder on academic performance. Gaultney (2010) conducted a study in North Carolina to explore the effect of sleep disorder on the academic performance of students and found that sleep disorders among students may have resulted in their academic failure. Hampton (2005) conducted a study in New Jersey and revealed that those students showed higher academic performances that have better sleep routines.

Generalizing from this information, scholars who are recurrently sleepy might be maximum their forthcoming possibilities in selecting calmer developments whereas in university. Thus, the occurrence of sleep disorders in university undergraduates hasn't been well-known. It is not acknowledged whether described drowsiness in university undergraduates is basically because of inadequate sleep or whether it may be unimportant to an undiagnosed sleep disorder. While described sleep disorders in grownups incline to proliferation with age, they might also happen in university students commonly sufficient to permit transmission of this populace. Furthermore, sleep outlines and disorders in university undergraduates might differ in gender or race/social association (Patel, Tran, Chakrabarti, Vasquez, Gilbert, \& Davidson, 2008). Based on conclusions, they decided that numerous university undergraduates are at danger for sleep disorders, as well as those at risk might also be at risk for educational failure.

\section{Methodology of the Study}

This study was quantitative in nature and the Survey method was used for data collection. The population of the study comprised of around 2000 students of BS from the Faculty of Social Sciences. The sample of the study consisted of 80 students who had reported that they suffer from sleep disorders to their respective hostel wardens. It was done through the purposive sampling technique by identifying the students with sleep disorders. The researcher used a self-developed three-point scale that was based on two major themes, which were reasons for sleep disorders \& the tendency of sleep disorder based on literature. It was on 3 points Likert scale. The validity of the instruments was determined through expert opinion and the instrument was modified accordingly. The reliability of the instruments was checked through Cronbach's Alpha and the calculated value was 0.75. The researcher personally visited all respondents for administering and getting backfilled questionnaires. The collected data were scored, tabulated, and analyzed making use of descriptive and inferential statistics i.e. mean scores and t-test. Data were analyzed through Statistical Package for Social Sciences (SPSS, 21).

Data Analysis and Findings

Tables were intended to understand the data as well as for further description.

Reasons of Sleep Disorder

Table 1

\begin{tabular}{llll}
\hline Scale & Always & Sometime & Never \\
\hline Computer, TikTok \& video games & $75 \%$ & $6.66 \%$ & $18.34 \%$ \\
Overnight use of cell phones & $46.66 \%$ & $21.67 \%$ & $31.67 \%$ \\
Use of energy drinks & $63.33 \%$ & $3.34 \%$ & $33.33 \%$ \\
Late-night socializing & $85 \%$ & $3.34 \%$ & $11.66 \%$ \\
Sleep environment not good & $61.66 \%$ & $30 \%$ & $8.34 \%$ \\
Stress-related to exam, assignment, and test & $85 \%$ & $3.34 \%$ & $11.66 \%$ \\
Homesickness & $50 \%$ & $30 \%$ & $20 \%$ \\
Eating heavy meal close to sleeping & $40 \%$ & $40 \%$ & $20 \%$ \\
Physical or mental disorder & $55 \%$ & $20 \%$ & $25 \%$ \\
Nightmares & $26.66 \%$ & $10 \%$ & $63.34 \%$ \\
\hline
\end{tabular}

Results of Table 1 reflected that stress related to exam, assignment/test, and late-night socializing were two major causes of students' sleep disorder as these causes have been reported by $85 \%$ of respondents. The second major cause was the use of computers, TikTok \& video games as $75 \%$ of respondents reported this reason. The third major cause was the use of energy drinks as $63 \%$ of responses were agreed against this statement. $61 \%$ stated that the sleep environment is not good, so that is stated as a fourth major cause of sleep disorder. Physical or mental disorder also is a major 
cause of sleep disorders and 55\% of students reported that problem. $50 \%$ of respondents stated that homesickness is "Always" a major cause of their sleep disorder. According to $46 \%$ of respondents, overnight use of cell phones is "Always" a major cause of their sleep disorder. $40 \%$ of respondents stated that due to eating heavy meals close to sleeping, they are facing the problem of sleep disorder. Nightmares are the least most cause of their sleep disorder, as only $26 \%$ of respondents stated this reason.

Showing Mean, Standard Deviation, Mean Difference, and t-value of students having High and Low-level sleep disorder

Based on collected data students were divided into two groups i.e. students having low-level sleep disorder and students having a high-level sleep disorder.

Table 2

Effect of sleep disorder on Academic Achievement

\begin{tabular}{llllll}
\hline Students & N & Mean & S.D & M.D & t-value \\
\hline Low-Level Sleep disorder & 40 & 67.52 & 16.86 & 9.36 & $2.47^{*}$ \\
High-level sleep disorder & 40 & 58.16 & 16.99 & & \\
\hline
\end{tabular}

The mean, S.D, and M.D of low and high-level sleep disorder in respect to academic achievement of students was found as $\mathrm{M}=67.52,58.16, \mathrm{~S} . \mathrm{D}=16.86,16.99$, and Mean difference=9.39 respectively. The obtained t-value of 2.47 was found more than the tabulated value (1.99) at 0.05 level of significance. Thus our findings suggest that students with low-level sleep disorder have high academic achievement than students with high-level sleep disorder based on our findings we can say our hypothesis (There is no effect of sleep disorders' on academic achievement of students) has been rejected.

\section{Discussion \& Conclusions}

Late-night socializing and stress related to exams, assignments, and tests were two top major causes of students sleep disorders. While using a computer, watching TikTok \& playing video games till late at night, use of energy drinks, uncomfortable sleep environment, and physical as well as mental disorders such as fever, headache, fatigue, stress and tension, etc. were regarded as second-level major causes of sleep disorder among students. Homesickness was regarded as the third level major cause of sleep disorder among students. Overnight use of cell phones eating a heavy meal close to sleeping was regarded as the fourth level major cause of sleep disorder among students. Results showed that there is a great effect of sleep disorders' on the academic achievement of students.

Results of this study have been also supported by Friedman (2003) \& Yang, et al. (2003) who stated based on their research studies that sleep disorders mainly affect student academic achievement. Furthermore, results are also supported by Gaultney (2010) who declared based on his research study that sleep disorder among students may have resulted in their academic failure. Students with low-level sleep disorder have high academic achievement than students having highlevel sleep disorder. Results of this study have been supported by other studies as well, such as Reisi et al. (2017) have also explored that sleep disorder has a negative effect on students' academic performance. Results are also aligned with the findings of Hampton (2005) who revealed that those students showed higher academic performance that have better sleep routines.

\section{Recommendations}

Recommendations of the study are: hostels' administrators may take adequate measures to reduce the adverse consequences of sleep disorder as it is affecting students' academic performance. Counseling facilities may be provided to students who are suffering from sleep disorders. Awareness seminars may be conducted for students regarding good sleep hygiene. Coordination among students, parents, and administrators may be ensured to improve the sleeping habits of students. Teachers may sensitize students that sleep is as essential for their health as food and water.

\section{References}

Anderson, B. Isser, A. \& Taylor, H. (2009). Associations of executive function with sleepiness and sleep duration in adolescents. Pediatrics; 123: e701-7.

Bao, Y., \& Yang, S. (2020, April). A Smartphone-based Sleep Monitoring Model. In 2020 AsiaPacific Conference on Image Processing, Electronics and Computers (IPEC) (pp. 449-454). IEEE.

Buysse, J. F. (2014). The prevalence of sleep disorders in college students: Impact on academic performance. The Journal of American College Health, 59, 91-97. 
Curcio, G. Ferrara, M. \& Gennaro, L. (2006). Sleep loss, learning capacity, and academic performance. Sleep Med Rev 2006; 10: 323- 37.

Dimitriou, D. Knight, F \& Milton, P. (2015). The Role of Environmental Factors on Sleep Patterns and School Performance in Adolescents. Front Psychol; 6: 1717.

Ebrahim, I. O., Shapiro, C. M., Williams, A. J., \& Fenwick, P. B. (2013). Alcohol and sleep I: Effects on normal sleep. Alcoholism: Clinical and Experimental Research, 37(4), 539-549.

Gomes, A. A., Tavares, J., \& de Azevedo, M. H. P. (2011). Sleep and academic performance in undergraduates: A multi-measure, multi-predictor approach. Chronobiology International, 28(9), 786-801.

Gaultney, J. (2010). The Prevalence of Sleep Disorders in College Students: Impact on Academic Performance. Journal of American College Health, 59, 2

Hampton, Tammy L. (2005). Impact of the lack of sleep on academic performance in college students. Masters' Dissertations.

Hershner, G., \& Chervin, J. (2014). The Impact of Sleep on Learning and Behavior in Adolescents. Teachers College Record, 104(4), 704-726.

Kelly, W. E., Kelly, K. E., \& Clanton, R. C. (2001). The relationship between sleep length and gradepoint average among college students. College Student Journal, 35(1), 84-86.

Lack, L. C. (1986). Delayed sleep and sleep loss in university students. Journal of American College Health, 35(3), 105-110. doi:10.1080/07448481.1986.9938970

McKim, K. (2007). Epidemiology of restless legs syndrome: the current status. Sleep Med Rev. 2006; 10:153-167.

Patel M, Tran D, Chakrabarti A, Vasquez A, Gilbert P \& Davidson, T. (2008). Prevalence of snoring in college students. $J$ Am Coll Health. 57:45-52.

Reisi M, Jalilian R, Azizi Gh, Rashti A, Faghihi nia J, Akbari M, et al. (2017). Academic Performance, Sleep Disorders and Their Association in Middle School Students in Iran. Int J Pediatr.

Torpey, E. (2015). Careers for night owls and early birds. Retrieved from http://www.bls.gov/ careeroutlook/2015/article/night-owls-and-earlybirds.htm\#for-more-information

Singleton, C. (2009) Adolescent sleep disturbance and school performance: The confounding variable of socioeconomics. Journal of Clinical Sleep Medicine, 3, 19.

Suchecki, R. B. \& Machado, P. A. (2009). Tiba, Stress-induced sleep rebound: adaptive behavior and possible mechanisms, Sleep Science, 2 (3), 151-160

Smith, L. (2001). Impact of Stress Factors on College Students Academic Performance. The University of North Carolina at Charlotte.

Wolfson, A. R. \& Carskadon, M. A. (2003). Understanding adolescents' sleep patterns and school performance: a critical appraisal. Sleep Med Rev; 7: 491-506.

Yang, C. M, Wu, C. H, Hsieh, M. H, Liu, M. H \& Lu F-W. (2003). coping with sleep disturbances among young adults: a survey of first-year college students in Taiwan. Behav Med. 29:133138.

Zeek, M. L., Savoie, M. J., Song, M., Kennemur, L. M., Qian, J., Jungnickel, P. W., \& Westrick, S. C. (2015). Sleep duration and academic performance among student pharmacists. American journal of pharmaceutical education, 79(5). 